\title{
Generalised transformer modelling for power flow calculation in multi-phase unbalanced networks
}

\author{
Massimiliano Coppo ${ }^{1} \bowtie$, Fabio Bignucolo ${ }^{1}$, Roberto Turri ${ }^{1}$ \\ ${ }^{1}$ Department of Industrial Engineering, University of Padova, via Gradenigo 6/A, Padova, Italy \\ 凶E-mail: massimiliano.coppo@unipd.it
}

\begin{abstract}
Low voltage systems are unbalanced networks where a significant share of the users is single-phase connected, so a multi-phase system needs to be considered in order to assess the mutual influence of the different phases. The presence of single-phase unevenly distributed users, leads to unbalances in the power flow on the three phases. This issue is emphasised considering the presence of local single-phase generators. This study presents a generalised method for transformers modelling in any multi-conductor grid representation in order to allow the analysis on unbalanced networks such as low-voltage distribution systems. The method, based on an incidence matrix approach, is proposed to represent any network object involving mutual connections among the phases, once the impedances for each single-phase equivalent circuit are known. Some application examples validate the approach and illustrate how to numerically realise the model.
\end{abstract}

\section{Introduction}

In the last few years, the intensification of renewable energy policies has led to a significant growth of distributed generation (DG) at the medium voltage (MV) and low voltage (LV) levels. In most of the cases, this has increased the penetration of single-phase DG units. For example, in the Italian case, LV networks host, at present, $>90 \%$ of the photovoltaic (PV) plants: almost 33\% are single phase with a rated power of $1-3 \mathrm{~kW}$, while $58 \%$ have a rated power of 3-20 kW, most of which are single phase [1]. From the ENTSO-E database on consumption by country [2], it can be seen that during summer the total load in the Italian grid can decrease up to about $30 \mathrm{GW}$ in the mid-day hours. This means that in those hours, assuming the PV units working at $80 \%$ of their rated power, about $10 \%$ of the demand is supplied by LV connected generators.

This fast changing scenario has prompted, for instance, a resolution by the Italian Authority for Electric Energy, Gas and Water (with the Italian acronym AEEGSI) in 2010 to foster smart grid projects and later [3] proposed a sort of criteria for the selection of projects based on different levels of smartness.

Since distribution systems were conceived as passive networks feeding a mainly balanced load, the presence of single-phase intermittent generators may increase the power flow unbalances. This may lead to possible issues not only to the users connected to the same LV grid, but to the upstream MV network as well. The provision of ancillary services to the distribution network supplied by microgrids in a market framework has been investigated in [4], highlighting the importance of a detailed analysis of the LV networks in the operation of modern power systems and evaluating the contribution of small scale generators to the network operation and safety. A study about the impact of those contributions in the ancillary services market is currently under way, considering the latest proposals by the Italian Authority [5]. Coordinated actions of LV connected active users [6] may improve the overall operating conditions of the entire distribution system, especially if coupled with a Distribution Management System at MV level [7-9].

For this reason, several research works were presented lately discussing the possible benefits coming from the connection of LV grids through On-Load-Tap-Changing (OLTC) transformers to mitigate the voltage unbalance. A Smart Transformer has been introduced in [10] to control the active power exchange between the main MV network and the LV grid where voltage-controlled inverters interface the DG units. Other works investigated the possibility of using specific transformer connections or even electronic transformers to deal with the voltage unbalance. The use of phase-decoupled OLTC capability of MV/LV transformers has been investigated in $[11,12]$ showing the possible improvement in the voltage controllability in LV networks. In [13], a balancing technique was proposed adopting a Scott transformer and a phaseangle regulating algorithm, while [14] discusses zig-zag transformers to attenuate the neutral current in three-phase fourwire distribution systems. In [15-17], a solid state transformer (SST) is presented as an interface capable of dealing with the bidirectional power flow typical of a smart grid environment, adding the Low-Voltage-Ride-Through and current unbalance management capabilities.

The need for a more reliable computation of the power flows in the distribution networks leads to the improvement of existing modelling and analysis methods, by considering multi-phase circuits in the power flow calculation. A review of existing methods and their recent developments was presented in [18], with the backward-forward sweep (BFS), the Newton-Raphson's family and the current injection methods being the most popular in distribution systems. Another effective method applied to the power flow computation in distribution networks is the $Z_{\text {bus }}$ Gauss method [19], even with unbalanced systems using the currentinjection technique $[20,21]$. A similar approach has been adopted by electric power research institute (EPRI) to develop OpenDSS, an open source multi-phase power flow tool [22].

Aiming to model multiple networks at different voltage levels, especially when the transformer is a fundamental part of the control scheme, it becomes necessary to represent in detail the mutual impedance between the network conductors, in order to evaluate the effects of regulation strategies over the entire system. Several works in the literature include the transformers in the power system by using reference-frame transformations. For instance, in [23] the $\alpha \beta 0$ stationary reference frame is adopted, while the sequence frame is used in [24]. A methodology to build the transformer admittance matrix directly in symmetrical components is proposed in [25], accounting for several connection schemes and phase shifting.

Although correctly representing the symmetrical system admittance, a more detailed definition of mutual coupling among the conductors is obtainable with the transformer model in the phase frame of reference as proposed in [26]. The concept was also adopted to develop multi-winding multi-terminal transformer models in [27-29]. An approach using the modified augmented nodal analysis is proposed in [30] to handle transformers in the 


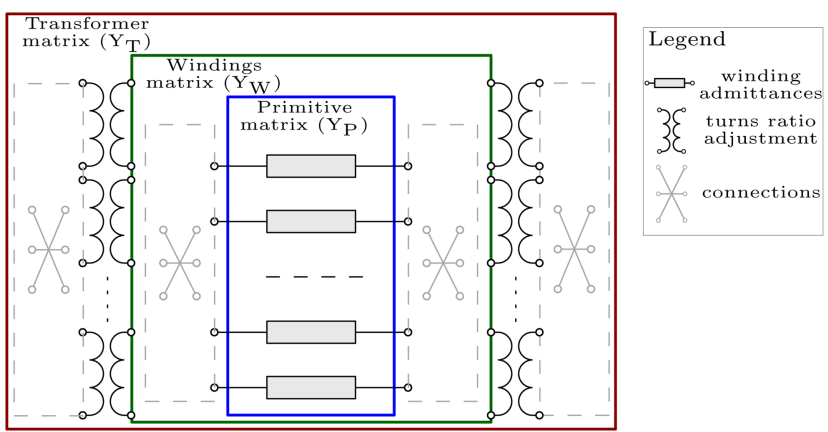

Fig. 1 Conceptual scheme of the construction methodology

BFS algorithm. Other works were presented to model particular transformer topologies for the connection of traction loads [31, 32].

Some of the works in the literature proposed approaches to model the transformer with balanced or unbalanced loading conditions, however, the proposed solutions either refer to specific cases or their extension to the general case is not clearly demonstrated. Through the method discussed in this paper, simple matrix operations are employed to represent any kind of transformer topology. The main advantage of this approach is the ease of customisation, since the same methodology can be applied to represent any branch element, including those with uneven number of phases, to support the implementation of new designs for the transformers and related control strategies in the power flow studies. The objective is to obtain a transformer model consistent with the multi-conductor power flow method presented in [33] adopting the concept of current-injection but also applicable to any other method based on the admittance matrix network representation. This paper is organised as follows: Section 2 illustrates the general modelling approach; in Section 3, the modelling methodology is applied to two-winding and threewinding transformers; Section 4 focuses on specific connection schemes, whereas Section 5 demonstrates how the proposed method is coherent with the existing approaches by illustrating a case study and validating the results obtained with those available in the literature. An additional validation is performed by applying the methodology to one of the IEEE test feeders in the Appendix.

\section{Modelling methodology}

In this section, the modelling approach for a transformer having a generic number of phases and windings is described. Arrays are indicated with bold capital letters, whereas dotted symbols stand for complex variables. Firstly, the circuit topology of a singlephase multi-winding transformer is defined, then the construction methodology for the multi-phase model is described by the following three sequential steps:

1. Definition of the transformer primitive admittance matrix $\boldsymbol{Y}_{\mathbf{P}}$.

2. Calculation of the (unconnected) windings admittance matrix $\boldsymbol{Y}_{\mathrm{W}}$.

3. Definition of the transformer admittance matrix $\boldsymbol{Y}_{\mathbf{T}}$ by applying the appropriate windings-ports connections to represent the topology and the group.

In Fig. 1, the aforementioned steps are pictorially shown in a conceptual scheme: it can be seen that the three admittance matrices introduced above can be considered as nested. Starting from the inner stage, where $\boldsymbol{Y}_{\mathbf{P}}$ is defined, each of the windings electrical properties are introduced, then second and third stages link them together to create the desired multi-phase circuit. Symbols in grey refer to connection stages, in which multi-phase circuit topologies are applied. The procedure described in the following makes use of the classical graph theory, commonly employed in power system modelling (see for instance [34]), applied to the multi-phase reference frame.

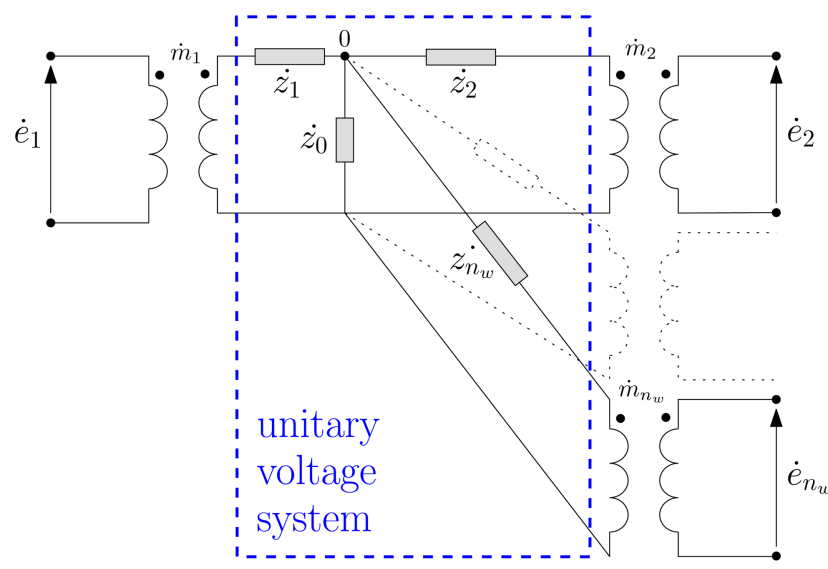

Fig. 2 Single-phase equivalent circuit of a multi-winding transformer

\subsection{Primitive admittance matrix $\boldsymbol{Y}_{\mathbf{P}}$}

The single-phase multi-winding transformer represents the elementary building block of any transformer model involving a generic number of windings and phases. This includes (as shown in the following sections) any type of standard and not standard transformer design.

The single-phase equivalent circuit in Fig. 2 is built by considering for each of the $n_{\mathrm{w}}$ windings identified by subscripts $1, \ldots, n_{\mathrm{w}}$ the respective impedance $\dot{z}_{1}, \ldots, \dot{z}_{n_{\mathrm{w}}}$, referred to a common voltage level here called unitary voltage system. The iron-core losses are taken into account by connecting the $\dot{z}_{0}$ impedance to the central node 0 . This model allows an exact representation of the transformer electrical characteristics since no approximations are required. The windings impedances are typically measured with short-circuit tests which could change depending on the number of windings involved. It is worth noting that the elementary building block is perfectly symmetrical if observed from each of the $n_{\mathrm{w}}$ ports.

In order to obtain a multi-phase transformer model, $n_{\mathrm{c}}$ singlephase circuits like the one in Fig. 2 need to be coupled. The admittances, obtained by inverting the impedance terms, can be collected in sub-matrices as in (1) for each winding and the shunt term is referred to the iron-core losses and the magnetic leakage. This leads to the unitary voltage system primitive admittance matrix $\boldsymbol{Y}_{\mathbf{P}_{(0)}}$ in (2). Subscript (0) indicates that the matrix explicitly refers to the central node 0

$$
\boldsymbol{Y}_{x}=\left[\begin{array}{ccc}
\frac{1}{\dot{z}_{x, a}} & 0 & 0 \\
0 & \ddots & 0 \\
0 & 0 & \frac{1}{\dot{z}_{x, n_{\mathrm{c}}}}
\end{array}\right]=\left[\begin{array}{ccc}
\dot{y}_{x, a} & 0 & 0 \\
0 & \ddots & 0 \\
0 & 0 & \dot{y}_{x, n_{\mathrm{c}}}
\end{array}\right], x=\left(1, \ldots, n_{\mathrm{w}}, 0\right.
$$

)

$$
\boldsymbol{Y}_{\mathbf{P}_{(0)}}=\left[\begin{array}{cccc}
\boldsymbol{Y}_{1} & \mathbf{0} & \mathbf{0} & \mathbf{0} \\
\mathbf{0} & \ddots & \mathbf{0} & \mathbf{0} \\
\mathbf{0} & \mathbf{0} & \boldsymbol{Y}_{\boldsymbol{n}_{\mathrm{w}}} & \mathbf{0} \\
\mathbf{0} & \mathbf{0} & \mathbf{0} & \boldsymbol{Y}_{0}
\end{array}\right]
$$

As it can be seen from (1), the order of each sub-matrix is given by the number of circuits $n_{\mathrm{c}}$, therefore the primitive admittance matrix in (2) $\boldsymbol{Y}_{\mathbf{P}_{(0)}}$ is a square matrix with order $\left[n_{\mathrm{c}} \cdot\left(n_{\mathrm{w}}+1\right)\right]$ since the number of included sub-matrices refers to each of the windings plus the shunt terms. 


\subsection{Windings admittance matrix $\boldsymbol{Y}_{\mathbf{W}}$}

In order to properly connect the admittances in each single-phase circuit, an admittance-winding incidence matrix $\boldsymbol{A}_{(0)}$ can be defined using identity and zeros matrices as shown in the following equation:

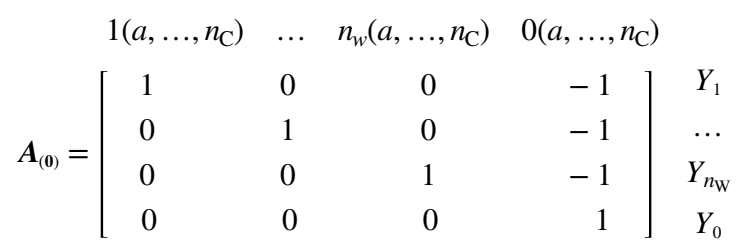

The column indices in (3) are grouped by winding (here indicated with $1, \ldots, n_{w}$ ) plus the explicit central node 0 and refer to the number of single-phase circuits in the model $n_{\mathrm{c}}$ (ports indicated as $\left.a, \ldots, n_{\mathrm{c}}\right)$. With this notation, identity and zeros sub-matrices $\mathbf{1}$ and 0 in (3) have order equal to the total number of ports $n_{\mathrm{c}}$, therefore $\boldsymbol{A}_{(0)}$ has order $\left[n_{\mathrm{c}} \cdot\left(n_{\mathrm{w}}+1\right)\right]$.

From (2) and (3), the windings admittance matrix for a multiphase transformer $\boldsymbol{Y}_{\mathbf{W}_{(0)}}$ is given by

$$
\boldsymbol{Y}_{\mathbf{W}_{(0)}}=A_{(0)}^{\mathrm{T}} \boldsymbol{Y}_{\mathbf{P}(0)} \boldsymbol{A}_{(0)}
$$

It is worth noting that this approach could be employed to represent a device connected to any number of busbars, each consisting of a generic number of phases. In fact, an additional winding could be included by just inserting the proper admittance terms in the diagonal of $\boldsymbol{Y}_{\mathbf{P}(0)}$ and adding blocks to $\boldsymbol{A}_{(0)}$ accordingly, while the number of the model phases could be changed by just modifying the number of $n_{\mathrm{c}}$ circuits.

Since the central node 0 should not be explicit in the transformer nodal admittance matrix (in order to include it in a power system model), a matrix reduction is performed through the process described by the following steps:

1. Invert $\boldsymbol{Y}_{\mathbf{W}_{(0)}}$ to obtain an impedance matrix of the order $\left[n_{\mathrm{c}} \cdot\left(n_{\mathrm{w}}+1\right)\right]$ which includes the explicit common node

$$
\boldsymbol{Z}_{\mathbf{W}(0)}=\left(\boldsymbol{Y}_{\mathbf{W}(0)}\right)^{-1}
$$

2. Delete rows and columns relative to the fictitious node, reducing the impedance matrix to order $\left[n_{\mathrm{W}} \cdot n_{\mathrm{c}}\right]: \boldsymbol{Z}_{\mathbf{W}(\mathbf{0})} \rightarrow \boldsymbol{Z}_{\mathrm{W}}$.

3. Invert $\boldsymbol{Z}_{\mathbf{W}}$ to get the reduced windings admittance matrix $\boldsymbol{Y}_{\mathbf{W}}$

$$
Y_{\mathbf{W}}=Z_{\mathbf{W}}^{-1}
$$

\subsection{Transformer admittance matrix $\boldsymbol{Y}_{\mathrm{T}}$}

The process described in the previous section allows to define the electrical characteristics of each single-phase circuit involved in the model but, in order to introduce the transformer in the power system model, the voltage rating at each side along with the ports mutual connections needs to be set, consequently defining the transformation ratio, topology and group.

Since the admittance terms are defined in a common unitary voltage system, a link with the external ports of the transformer, represented as ideal couplings in Fig. 2, is introduced through the per-unit turns ratio terms $\dot{m}_{w_{\mathrm{c}}}$ (with $w=1, \ldots, n_{\mathrm{w}}$ and $c=1, \ldots, n_{\mathrm{c}}$ ) and is defined as

$$
\dot{m}_{w_{\mathrm{c}}}=1 /\left[\left(\dot{E}_{w_{\mathrm{c}}}+\Delta \dot{E}_{w_{\mathrm{c}}}\right) / E_{w_{\mathrm{c}} \mathrm{Base}}\right]=1 /\left(\dot{e}_{w_{\mathrm{c}}}+\Delta \dot{e}_{w_{\mathrm{c}}}\right)
$$

where subscripts $c$ and $w$ identify the circuit and the winding, respectively, while $\dot{E}_{w_{\mathrm{c}}}$ and $E_{w_{\mathrm{c}} \text { Base }}$ are, for winding $w$, the circuit rated voltage and the base voltage, respectively. The $\Delta \dot{e}_{w_{\mathrm{c}}}$ term introduces a variation to the turns ratio due to a possible control action (e.g. tap changer). It is worth noting that the $\Delta \dot{e}_{w_{\mathrm{c}}}$, and consequently the per-unit turns ratio terms, could be either integer or complex (in case the control action requires either a turns ratio modulation or a rotation of the voltage phasors).

The terms defined as in (7) are then stored in the matrix $\boldsymbol{M}$ which allows a straight forward application of the turns ratios to the windings admittance matrix

$$
\boldsymbol{M}=\left[\begin{array}{ccccccc}
\dot{m}_{1_{a}} & 0 & 0 & 0 & 0 & 0 & 0 \\
0 & \ddots & 0 & \ldots & \ldots & \ldots & 0 \\
0 & 0 & \dot{m}_{1_{n_{c}}} & 0 & \ldots & \ldots & 0 \\
0 & \vdots & 0 & \ddots & 0 & \ldots & 0 \\
0 & \vdots & \vdots & 0 & \dot{m}_{n_{w_{a}}} & 0 & 0 \\
0 & \vdots & \vdots & \vdots & 0 & \ddots & 0 \\
0 & 0 & 0 & 0 & 0 & 0 & \dot{m}_{n_{w_{\mathrm{c}}}}
\end{array}\right]
$$

Along with the turns ratio information introduced by $\boldsymbol{M}$, the topology of the external connections can be set by defining a suitable winding-port incidence matrix $\boldsymbol{C}$ as shown in the following equation:

$$
C=\left[\begin{array}{ccc}
C_{1} & 0 & 0 \\
0 & \ddots & 0 \\
0 & 0 & C_{n_{\mathrm{w}}}
\end{array}\right]
$$

where $\boldsymbol{C}_{\left(\mathbf{1}, \ldots, n_{\mathrm{w}}\right)}$ sub-matrices contain the ports connection topology for each winding, having the number of rows equal to the number of single-phase circuits $n_{\mathrm{c}}$ and columns dimension given by the number of ports in the power system model to which the transformer is going to be connected. For example, if the external busbar to which winding 1 is connected is modelled as a threephase plus neutral bus, $\boldsymbol{C}_{1}$ has four columns. Considering the number of ports of each side defined as $p_{1}, \ldots, p_{n_{\mathrm{w}}}$, the dimension of $\boldsymbol{C}$ becomes [ $\left.\left(n_{\mathrm{w}} \cdot n_{\mathrm{c}}\right) \times\left(p_{1}+\cdots+p_{n_{\mathrm{w}}}\right)\right]$.

The transformer admittance matrix $\boldsymbol{Y}_{\mathbf{T}}$ for the multi-phase multi-winding transformer is finally obtained as

$$
\boldsymbol{Y}_{\mathrm{T}}=\boldsymbol{C}^{\mathrm{T}}\left\{\boldsymbol{M} \boldsymbol{Y}_{\mathrm{W}} \boldsymbol{M}\right\} \boldsymbol{C}
$$

\section{Modelling of standard three-phase transformers}

In this section, the generalised modelling methodology is applied to represent standard three-phase constructions such as the twowinding and three-winding transformers. The aim is to demonstrate the high flexibility in the topology definition by just modifying the incidence matrices.

\subsection{Three-phase two-winding transformer}

This category of transformers is widespread in power systems and consists of two sides (primary and secondary), each with 3 or 4 ports depending if the neutral node is explicitly represented or not.

In Fig. 3, a delta-wye transformer circuit is depicted and the three steps illustrated in the previous section can be identified in the scheme: the unitary voltage system (in which the impedances are defined) is connected through ideal couplings (setting the turn ratios) to the ports which are suitably connected to reproduce the transformer topology.

The impedances related to each of the windings phases are generally measured with short-circuit and no-load tests. For a standard three-phase transformer, the measured impedances are defined in terms of magnitude as shown in the following equations:

$$
\begin{gathered}
\left|\dot{z}_{\mathrm{scx}}\right|=v_{\mathrm{sc}} \cdot \frac{S_{\text {base }}}{S_{n x}} \\
\left|\dot{z}_{0 x}\right|=\frac{1}{\left|\dot{y}_{0 x}\right|}=\left(i_{0} \cdot \frac{S_{n x}}{S_{\text {base }}}\right)^{-1}
\end{gathered}
$$




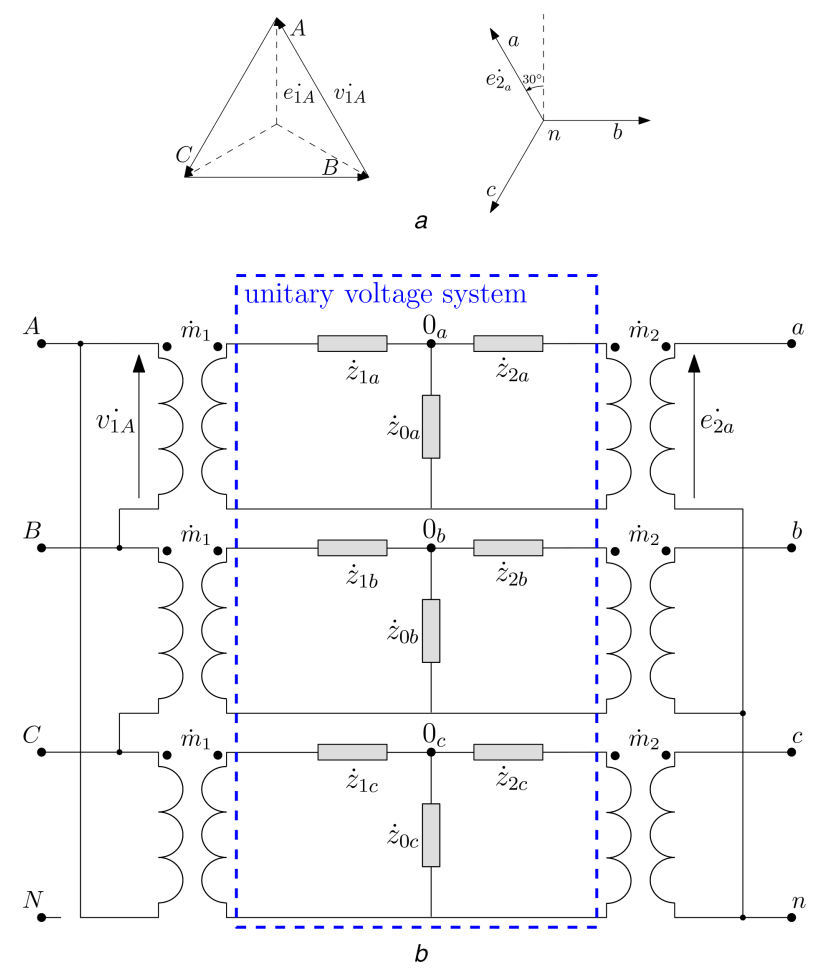

Fig. 3 Three-phase delta-wye transformer

(a) Vectorial diagram and (b) Connection scheme

where $\dot{z}_{\mathrm{sc}}$ is the short-circuit impedance of phase $x, v_{\mathrm{sc}}$ is the feeding voltage in the short-circuit test in p.u., $i_{0}$ is the feeding current in the open-circuit test in p.u., $S_{n x}$ is the rated power of each single-phase circuit and $S_{\text {base }}$ is the system base power. Impedances $\dot{z}_{\text {sc }}$ and $\dot{z}_{0}$ can be represented as complex values according to the measured active power absorption in the short-circuit test and noload test, respectively. In the case of two-winding transformers, relation (13) highlights how each winding impedance is defined as a share of the measured value. Generally, $\dot{z}_{1 x}$ is considered half of $\dot{z}_{\text {sc } x}$ (in p.u.), even if other splitting rules could be considered (e.g. if winding DC resistance is known). If $\dot{z}_{2 x}$ is supposed to be equal to $\dot{z}_{\mathrm{sc}}$, the frequently used transformer equivalent model is obtained, allowing a simplified computation of no-load absorption and transformer voltage drop

$$
\left|\dot{z}_{\mathrm{sc} x}\right|=\left|\dot{z}_{1 x}+\dot{z}_{2 x}\right|
$$

The primitive admittance matrix is built as shown in Section 2.1 with $n_{\mathrm{w}}=2$ and $n_{\mathrm{c}}=3$. It should be noted that if both $z_{1 x}$ and $z_{2 x}$ are defined as half of $z_{\mathrm{sc}}$, the terms included in each of the diagonal sub-matrices $\boldsymbol{Y}_{1}$ and $\boldsymbol{Y}_{2}$ are all equal. Each of the winding primitive sub-matrices is of order 3 so the order of matrices $\boldsymbol{Y}_{\mathbf{P}(0)}, \boldsymbol{A}_{(0)}$ and $\boldsymbol{Y}_{\mathbf{W}_{(0)}}$ is 9 .

After the process described in Section 2.2, the windings matrix $\boldsymbol{Y}_{\mathbf{W}}$ is square with order 6 and the transformer admittance matrix $\boldsymbol{Y}_{\mathrm{T}}$ can be obtained using suitably defined matrices $\boldsymbol{M}$ and $\boldsymbol{C}$ which set the turns ratio and connection topology, respectively. Considering the example in Fig. $3 b$ (delta-wye transformer) and having chosen the phase-neutral voltages $e_{1}$ and $e_{2}$ as base values on each side, respectively, according to (7) the turn ratios $m_{1}$ and $m_{2}$ result as

$$
\left\{\begin{array}{l}
\dot{m}_{1}=1 / \dot{v}_{1}=1 /\left(\sqrt{3} \dot{e}_{1}\right)=1 / \sqrt{3} \\
\dot{m}_{2}=1 / \dot{e}_{2}=1
\end{array}\right.
$$

In order to set the connections among the external ports, the incidence matrix $\boldsymbol{C}$ has to be defined. As it could be noticed from Fig. $3 b$, all the ports of a three-phase system are generically considered (including the neutral on each side) to represent any

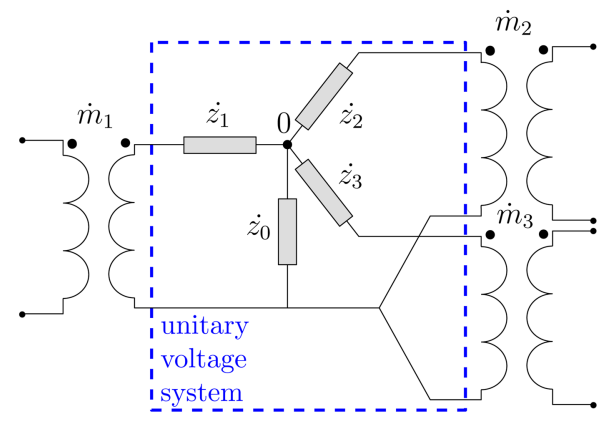

Fig. 4 Elementary single-phase equivalent circuit of a three-winding transformer

possible coupling between each port and the external system (e.g. the earth potential). Considering the entire network in which the transformer is inserted, in case one of the ports has no interactions with the system, it has to be eliminated from the model (by reducing the number of columns) to avoid matrix inversion issues. For the three-phase circuit in Fig. 3b, having four external ports on each side and three elementary circuits, the connection matrix is built as in (15) defining two sub-matrices $\boldsymbol{C}_{1}$ and $\boldsymbol{C}_{2}$, each with dimensions $[3 \times 4]$. In this case, the neutral port is explicit on the primary side, even though it has no interaction with the transformer, resulting in a zeros column, to show how it can be included in the model to be consistent with the number of phases in the external system

$$
\begin{aligned}
& \begin{array}{llllllll}
A & B & C & N & a & b & c & n
\end{array} \\
& \boldsymbol{C}=\left[\begin{array}{cccccccc}
1 & -1 & 0 & 0 & 0 & 0 & 0 & 0 \\
0 & 1 & -1 & 0 & 0 & 0 & 0 & 0 \\
-1 & 0 & 1 & 0 & 0 & 0 & 0 & 0 \\
0 & 0 & 0 & 0 & 1 & 0 & 0 & -1 \\
0 & 0 & 0 & 0 & 0 & 1 & 0 & -1 \\
0 & 0 & 0 & 0 & 0 & 0 & 0 & -1
\end{array}\right] \begin{array}{l}
A-B \\
B-C \\
C-A \\
a-n \\
b-n \\
c-n
\end{array}=\left[\begin{array}{cc}
C_{1} & \mathbf{0} \\
\mathbf{0} & C_{2}
\end{array}\right](15)
\end{aligned}
$$

$\boldsymbol{C}_{\mathbf{1}}$ is the typical incidence sub-matrix representing a delta connection between windings (the last column, composed by zeros, has to be deleted in case the port $N$ is not defined in the network model as in three-wire neutral-isolated distribution networks). Similarly, $\boldsymbol{C}_{2}$ is the typical incidence sub-matrix referred to the wye connection of the windings. It should be noted that the transformer topology resulting from the application of the $\boldsymbol{C}$ matrix also defines the transformer group (in this case 11), since it involves a phase displacement between the primary and secondary phase-voltages as shown from the vectorial diagram in Fig. $3 a$.

\subsection{Three-phase three-winding transformer}

In the following, the case of a three-phase three-winding transformer is considered as an example of a device connected to multiple busbars, each consisting of a generic number of phases. The modelling approach described in Section 2 can be easily applied once the impedances in the elementary single-phase circuits are defined with reference to the topology shown in Fig. 4.

In this case, the short-circuit impedances $\dot{z}_{\mathrm{sc1} 2}, \dot{z}_{\mathrm{sc} 23}$ and $\dot{z}_{\mathrm{sc1} 13}$ can be obtained from experimental tests for each pair of buses indicated by the respective subscript. Standing the currently applied measurement procedure for a three-winding transformer, the short-circuit impedance between each couple of sides is calculated supplying the transformer from one side, with another one short-circuited side and the third left open. For example, $\dot{z}_{\mathrm{sc}_{12}}$ is measured supplying bus 1 , short-circuiting bus 2 and leaving bus 3 open. In this way, each experimentally measured short-circuit impedance is equal to the vectorial sum of two of the three terms $\dot{z}_{1}, \dot{z}_{2}, \dot{z}_{3}$ in Fig. 4. Putting this concept in matrix form, the three aforementioned terms are defined as follows: 


$$
\left[\begin{array}{l}
\dot{z}_{1} \\
\dot{z}_{2} \\
\dot{z}_{3}
\end{array}\right]=\frac{1}{2}\left[\begin{array}{ccc}
1 & -1 & 1 \\
1 & 1 & -1 \\
-1 & 1 & 1
\end{array}\right] \cdot\left[\begin{array}{l}
\dot{z}_{\mathrm{sc} 12} \\
\dot{z}_{\mathrm{sc} 23} \\
\dot{z}_{\mathrm{sc} 13}
\end{array}\right]
$$

After inversion, the impedances defined in (16) can be grouped separately for the three sides along with the shunt terms referred to the magnetising losses, which leads to the primitive admittance matrix shown in compact form as follows:

$$
\boldsymbol{Y}_{\mathbf{P}(0)}=\left[\begin{array}{cccc}
\boldsymbol{Y}_{1} & \mathbf{0} & \mathbf{0} & \mathbf{0} \\
\mathbf{0} & \boldsymbol{Y}_{2} & \mathbf{0} & \mathbf{0} \\
\mathbf{0} & \mathbf{0} & \boldsymbol{Y}_{3} & \mathbf{0} \\
\mathbf{0} & \mathbf{0} & \mathbf{0} & \boldsymbol{Y}_{0}
\end{array}\right]
$$

Similar to the previous section, $\boldsymbol{Y}_{\mathbf{P}(0)}$ is built by diagonal submatrices with order $n_{\mathrm{c}}=3$, so the result is a square matrix with order 12. The methodology described in Section 2 for the general case can be easily applied in this case too, so the order of matrices $\boldsymbol{A}_{(0)}$ and $\boldsymbol{Y}_{\mathbf{W}_{(0)}}$ is 12, while $\boldsymbol{Y}_{\mathbf{W}}$ and $\boldsymbol{M}$ are of order 9. The assumptions made previously for the transformation ratio and topology setting, to define matrices $\boldsymbol{M}$ and $\boldsymbol{C}$, are still valid for each winding depending on the kind of connection to the external power system in each bus. For the standard delta and wye topologies, the same values introduced in (14) and the sub-matrices used in (15) can be applied to define each of the windings connection to the external ports.

\section{Examples of non-standard transformers modelling}

In the previous sections, the method for modelling transformers with any number of phases and windings has been presented and applied to represent standard transformers used in power systems. In the following, the zig-zag and the Scott connections are considered as particular application examples of the proposed generalised method.

\subsection{Zig-zag connection}

This kind of connection is typically used to create a common reference for the neutral point in three-phase/three-wire systems or, in general, to reduce the amount of voltage unbalance on the primary side while feeding an unbalanced load. Voltage diagrams of the primary and secondary sides are shown in Fig. $5 a$ which refer to the circuit depicted in Fig. $5 b$.

As in the case of a two-winding transformer discussed in Section 3.1, the impedance related to each side can be obtained as a share of the short-circuit impedance (typically half) with the introduction of the central node 0 . Then, the same considerations for calculating the admittance matrix are valid. Choosing the rated phase-neutral voltages as base values on each side and considering the vectorial diagram in Fig. $5 a$, (7) yields the turn ratios for the two sides

$$
\left\{\begin{array}{l}
\dot{m}_{1}=1 / \dot{e}_{1}=1 \\
\dot{m}_{2}=1 / \dot{e}_{2}=2 / \sqrt{3}
\end{array}\right.
$$

Considering the vectorial diagram and the three-phase circuit shown in Fig. 5, the incidence matrix of a wye-zig-zag transformer with group 11 can be built as in (15) with the sub-matrices defined as
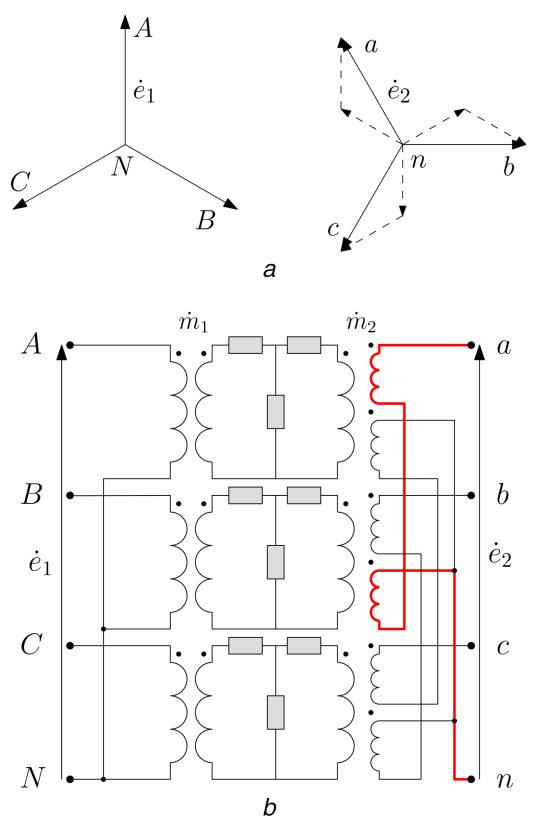

Fig. 5 Three-phase wye-zig-zag transformer

(a) Vectorial diagram and (b) Connection scheme. The path for the phase-neutral voltage in phase $a$ is highlighted on the secondary side

$$
\begin{aligned}
\boldsymbol{C}_{1} & =\left[\begin{array}{llll}
1 & 0 & 0 & -1 \\
0 & 1 & 0 & -1 \\
0 & 0 & 1 & -1
\end{array}\right] \\
\boldsymbol{C}_{2} & =\left[\begin{array}{ccccc}
r & -(1-r) & 0 & 0 \\
0 & r & -(1-r) & 0 \\
-(1-r) & 0 & r & 0
\end{array}\right] \\
& =\left[\begin{array}{cccc}
0.5 & -0.5 & 0 & 0 \\
0 & 0.5 & -0.5 & 0 \\
-0.5 & 0 & 0.5 & 0
\end{array}\right]
\end{aligned}
$$

As could be seen from the vectorial diagram, the secondary voltage is obtained as a composition of two half-winding voltages on the different phases. To include this aspect in the incidence matrix definition, the connections among the phases have been set using the parameter $r$ as shown in (19) with $r=0.5$. According to Section 3.1, the last column of $\boldsymbol{C}_{2}$ has not to be deleted if the secondary side of the wye-zig-zag transformer includes the neutral point (e.g. to connect a Petersen coil).

\subsection{Scott transformer}

The Scott transformer is employed for the connection of traction systems (which require two-phases displaced by $90^{\circ}$ ) to a threephase network, aiming at reducing the amount of unbalance on the three-phase grid due to the asymmetrical nature of the traction system. This kind of connection can be represented through the general approach in Section 2 by simply setting a suitable incidence matrix defining the interaction between the windings, based on the voltage vectors composition shown in Fig. $6 a$. Fig. $6 b$ reports the internal connection scheme of the Scott transformer $\left(n_{\mathrm{c}}=2, n_{\mathrm{w}}=2\right)$.

As for the zig-zag connection, one of the windings (here on the primary side) is divided in two half-windings, so the incidence matrix involves fractional terms. In this way, the voltage vectors applied to the primary side ports shown in Fig. $6 a$ are obtained as a combination of the phase potentials. Furthermore, since the number of phases on the two sides is different, $\boldsymbol{C}_{1}$ and $\boldsymbol{C}_{2}$ sub-matrices have uneven dimensions, respectively, [2 $2 \times 4]$ and [ $2 \times 3]$ 

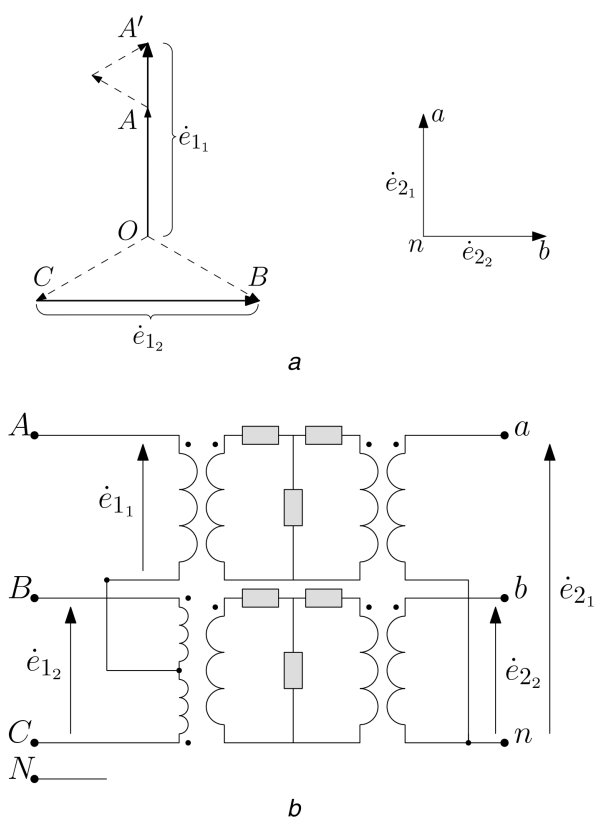

Fig. 6 Scott transformer

(a) Vectorial diagram and (b) Connection scheme. The vector composition of phase voltages is highlighted for the primary side

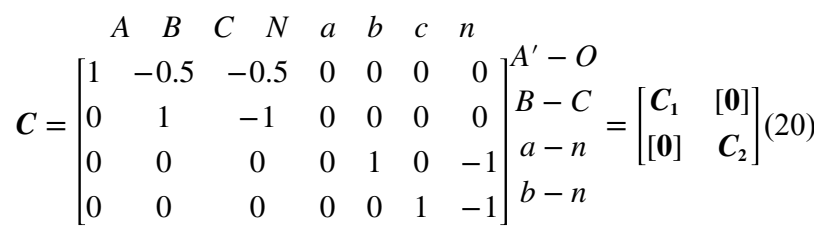

From (20) it can be seen that the number of phases on the primary side is still kept as four with the explicit neutral node, confirming that the number of phases can be chosen independently from the ports actually used in the model. In this case, as discussed in Section 3.1, the neutral point needs to be connected to earth through an external impedance (i.e. neutral point earthing) to avoid inversion problems when including the transformer model in the system admittance matrix. Alternatively, if the neutral point is not represented in the network model on the three-phase side, $\boldsymbol{C}_{\boldsymbol{1}}$ dimension has to be reduced to [ $2 \times 3]$ by deleting its last column.

Unlike the previous examples, not only the turns ratio change from one side to the other, but also from one phase-winding to the other. Following the approach described in Section 2.3, the matrix $\boldsymbol{M}$ sets the turns ratio for each elementary circuit, by selecting the base voltages as the rated phase-neutral values:

$$
\boldsymbol{M}=\left[\begin{array}{cccc}
1.5 & 0 & 0 & 0 \\
0 & \sqrt{3} & 0 & 0 \\
0 & 0 & 1 & 0 \\
0 & 0 & 0 & 1
\end{array}\right]
$$

\section{Case study application}

In this section, an application of the proposed approach for the transformer modelling is presented to compare different connection options dealing with the load unbalance. This kind of analysis has been employed in [31] where the authors applied a different solution method and thus can be taken as a useful reference for testing the proposed generalised modelling procedure. An additional validation of the presented generalised approach is performed by applying it to the IEEE 4-bus Test Feeder Case [35].

The case study is shown in Fig. $7 a$ the main three-phase network feeding the transformer has been modelled as a series of an ideal three-phase voltage source (composed by three ideal wyeconnected voltage generators at Bus 1) in series with the longitudinal impedances $z_{\mathrm{s}}$. Loads are connected to the secondary
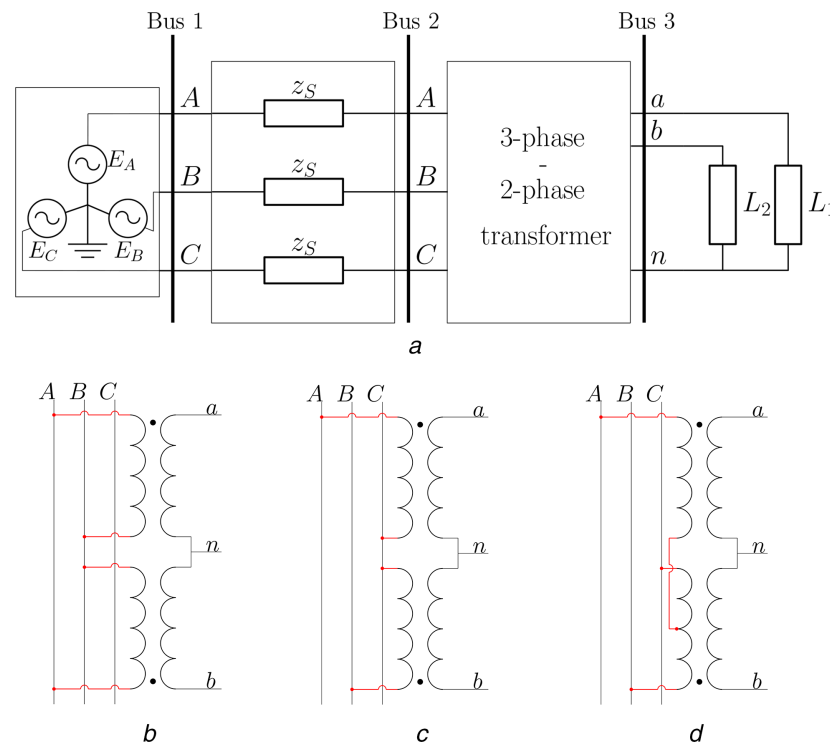

Fig. 7 Main three-phase network feeding the transformer

(a) Equivalent circuit used for the proposed approach validation. Different topology options are compared for the three-phase/two-phase transformer model to test the effects of different load unbalances: (b) Single phase, (c) V connection, (d) Scott connection

side of the transformer (Bus 3) in a two-phase plus neutral system as in the case of a traction load.

In the following, the method described in the previous sections is applied to model a three-phase/two-phase traction transformer with three different connection options. Figs. $7 b-d$ show the topological schemes of the three options considered for connecting Buses 2 to 3. It should be noted that, since in [31] no interactions between the neutral and the system are defined, in this application the transformer model presents three ports on both the primary $\left(p_{1}=3\right)$ and secondary $\left(p_{2}=3\right)$ sides. As it can be seen in Fig. $7 a$ the transformer is composed by two single-phase circuits $\left(n_{\mathrm{c}}=2\right)$. As a result, the dimension of the corresponding incidence matrix $C$ is $[(2 \times 2) \times(3+3)]=[4 \times 6]$. Its composition for the three cases, respectively, is shown in (22). The third column of matrix $\boldsymbol{C}_{\mathbf{1 p h}}$ has not to be deleted since phase $C$ is defined in the system, even if it is not connected to the transformer is this particular scenario

$$
\begin{aligned}
\boldsymbol{C}_{\mathbf{1 p h}} & =\left[\begin{array}{cccccc}
1 & -1 & 0 & 0 & 0 & 0 \\
1 & -1 & 0 & 0 & 0 & 0 \\
0 & 0 & 0 & 1 & 0 & -1 \\
0 & 0 & 0 & 0 & 1 & -1
\end{array}\right] \\
\boldsymbol{C}_{\mathbf{V}-\text { conn }} & =\left[\begin{array}{cccccc}
1 & 0 & -1 & 0 & 0 & 0 \\
0 & 1 & -1 & 0 & 0 & 0 \\
0 & 0 & 0 & 1 & 0 & -1 \\
0 & 0 & 0 & 0 & 1 & -1
\end{array}\right] \\
\boldsymbol{C}_{\text {Scott }} & =\left[\begin{array}{cccccc}
1 & -0.5 & -0.5 & 0 & 0 & 0 \\
0 & 1 & -1 & 0 & 0 & 0 \\
0 & 0 & 0 & 1 & 0 & -1 \\
0 & 0 & 0 & 0 & 1 & -1
\end{array}\right]
\end{aligned}
$$

\subsection{Test circuit data}

The data for the test circuit shown in Fig. $7 a$ are taken from [31] and are recalled in the following. An ideal three-phase voltage source is connected to Bus 1, operated as a slack bus with the phase-voltages as follows:

$$
\left\{\begin{array}{l}
E_{A}=E_{\mathrm{LN} s} \cdot \exp ^{\mathrm{j} 0} \\
E_{B}=E_{\mathrm{LN} s} \cdot \exp ^{\mathrm{j}(-2 \pi / 3)} \\
E_{C}=E_{\mathrm{LN} s} \cdot \exp ^{\mathrm{j}(2 \pi / 3)}
\end{array}\right.
$$



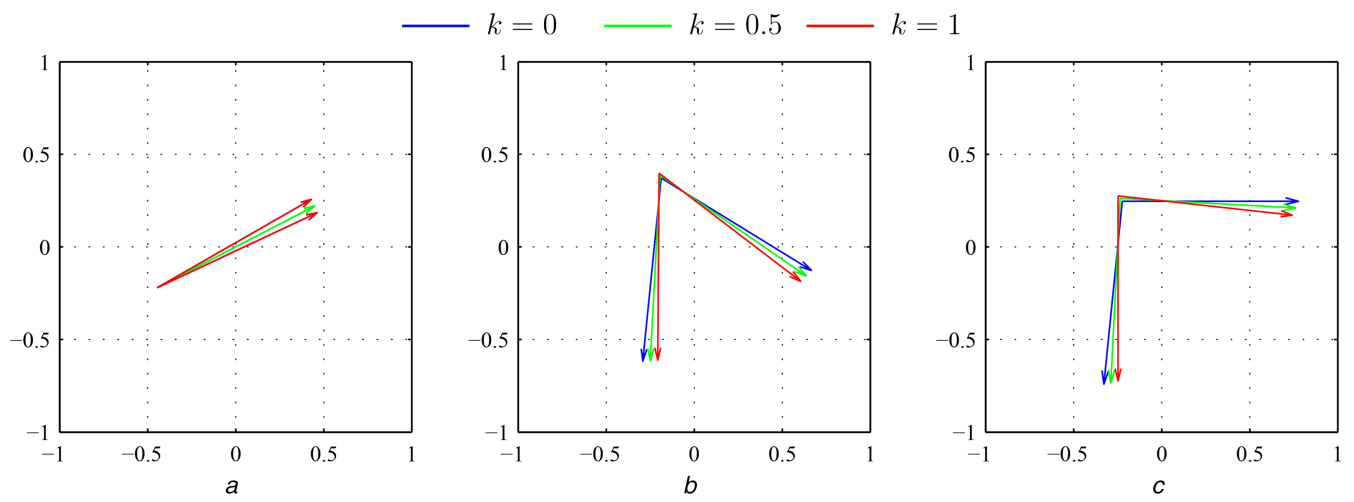

Fig. 8 Phase voltage on the load bus with different connection options and isolated neutral under three load unbalance conditions: $k=0$ (blue), $k=0.5$ (green) and $k=1$ (red)

(a) Single-phase connection, (b) V connection, (c) Scott connection

Table 1 Phase voltages under three unbalance conditions with each connection option

\begin{tabular}{|c|c|c|c|c|c|c|c|}
\hline Conn. & $k$ & $\begin{array}{c}E_{A B} \\
\mid \text { p.u.| } \angle 0\end{array}$ & $\begin{array}{c}E_{B C} \\
\mid \text { p.u.| } \angle 0\end{array}$ & $\begin{array}{c}E_{C A} \\
\mid \text { p.u.| } \angle^{\circ}\end{array}$ & $\begin{array}{c}E_{a n} \\
\mid \text { p.u. } \mid \angle 0\end{array}$ & $\begin{array}{c}E_{b n} \\
\mid \text { p.u.| } \angle^{\circ}\end{array}$ & $\begin{array}{c}E_{n} \\
\mid \text { p.u.| } \angle 0\end{array}$ \\
\hline \multirow[t]{3}{*}{ 1-ph } & 0.0 & $1.728 \angle 28.6$ & $1.712 \angle-90.3$ & $1.750 \angle 149.6$ & $0.998 \angle 28.6$ & $0.995 \angle 24.0$ & $0.498<-153.7$ \\
\hline & 0.5 & $1.730 \angle 28.6$ & $1.713 \angle-90.3$ & $1.750 \angle 149.6$ & $0.998 \angle 26.3$ & $0.998 \angle 26.3$ & $0.499 \angle-153.7$ \\
\hline & 1.0 & $1.728 \angle 28.6$ & $1.712 \angle-90.3$ & $1.750 \angle 149.6$ & $0.995 \angle 24.0$ & $0.998<28.6$ & $0.498<-153.7$ \\
\hline \multirow[t]{3}{*}{ V } & 0.0 & $1.750 \angle 29.6$ & $1.728 \angle-91.4$ & $1.712 \angle 149.7$ & $0.989 \angle-30.3$ & $0.995 \angle-96.0$ & $0.417 \angle 116.7$ \\
\hline & 0.5 & $1.732 \angle 29.6$ & $1.740<-90.9$ & $1.721 \angle 149.1$ & $0.993 \angle-33.2$ & $1.004 \angle-93.2$ & $0.432 \angle 116.6$ \\
\hline & 1.0 & $1.712 \angle 29.7$ & $1.750 \angle-90.4$ & $1.728 \angle 148.6$ & $0.995 \angle-36.0$ & $1.010 \angle-90.4$ & $0.446 \angle 116.6$ \\
\hline \multirow[t]{3}{*}{ Scott } & 0.0 & $1.750 \angle 29.6$ & $1.728<-91.4$ & $1.712 \angle 149.7$ & $1.000 \angle 0.0$ & $0.995 \angle-96.0$ & $0.334 \angle 132.2$ \\
\hline & 0.5 & $1.731 \angle 29.3$ & $1.731 \angle-90.7$ & $1.731 \angle 149.3$ & $0.999 \angle-3.0$ & $0.999 \angle-93.0$ & $0.353 \angle 132.0$ \\
\hline & 1.0 & $1.710 \angle 29.0$ & $1.732 \angle-90.0$ & $1.748 \angle 148.9$ & $0.995 \angle-6.0$ & $1.000 \angle-90.0$ & $0.371<131.9$ \\
\hline
\end{tabular}

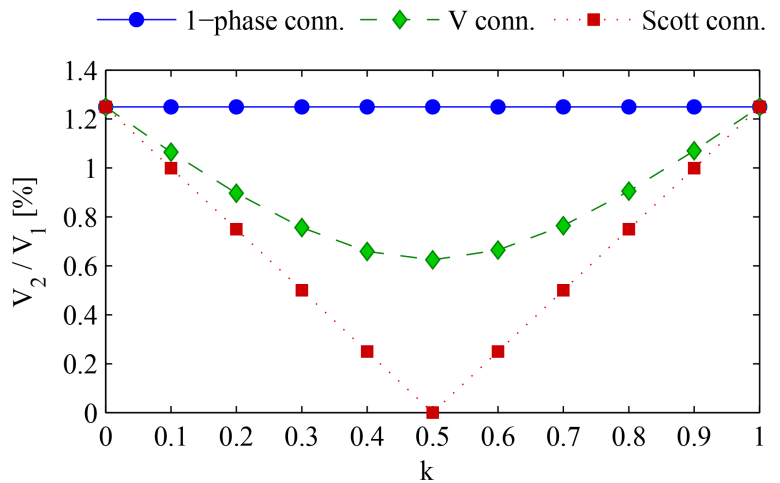

Fig. 9 Comparison among the three different kinds of connections in relation to the load unbalance. The ratio between the negative and positive sequence components is used to report the voltage unbalance on the primary side

where $E_{\mathrm{LN} s}$ is the line-to-neutral nominal voltage magnitude. Defining the base values for the apparent power and the voltage as $S_{\text {Base }}$ and $E_{\text {Base }}$ (phase values), respectively, the magnitude of the impedance $\dot{z}_{S}$ for each phase (assumed purely inductive) is calculated as

$$
z_{\mathrm{S}}=\left|\dot{z}_{\mathrm{S}}\right|=\frac{V_{\mathrm{LL} s}^{2}}{S_{\mathrm{S}}} \cdot \frac{S_{\text {Base }}}{E_{\text {Base }}^{2}} \mathrm{p} \cdot \mathrm{u}
$$

where $S_{\mathrm{S}}$ is the source three-phase short-circuit apparent power and $V_{\mathrm{LL} s}$ is the source line-to-line voltage $\left(V_{\mathrm{LL} s}=\sqrt{3} E_{\mathrm{LN} s}\right)$.

The magnitude of the transformer short-circuit impedance $z_{\mathrm{T}}$ (in [31] supposed purely inductive) is calculated as

$$
z_{\mathrm{T}}=\left|\dot{z}_{\mathrm{T}}\right|=\frac{E_{\mathrm{sc}}}{E_{1 \mathrm{R}}} \cdot \frac{E_{1 \mathrm{R}}^{2}}{S_{\mathrm{R}}} \cdot \frac{S_{\text {Base }}}{E_{\text {Base }}^{2}} \mathrm{p} \cdot \mathrm{u} .
$$

where $E_{\mathrm{sc}}$ is the short-circuit voltage in $\mathrm{V}, E_{\mathrm{lR}}$ is the rated voltage at the primary side in $\mathrm{V}$ and $S_{\mathrm{R}}$ is the rated apparent power for one phase in VA.

Finally, load impedances are defined as in (26), depending on the absorbed power:

$$
z_{\mathrm{L} x}=\frac{E_{\mathrm{L} N l}^{2}}{S_{\mathrm{L} x}} \cdot \frac{S_{\text {Base }}}{E_{\text {Base }}^{2}} \quad(x=1,2), \mathrm{p} \cdot \mathrm{u} .
$$

where $E_{\mathrm{L} N l}$ is the load phase voltage in $\mathrm{V}$ and $S_{\mathrm{L} x}$ is the $x$ th singlephase load power in $\mathrm{W}$ (since loads are considered to have unitary power factor).

In this work, assuming $S_{\text {Base }}$ as the transformer single-phase rated power $\left(S_{\mathrm{R}}\right)$ and $E_{\mathrm{Base}}$ as the phase-neutral voltage of the source $\left(E_{\mathrm{LNS}}\right)$, the magnitudes of the phase voltages in (23) become 1 p.u. Impedances are calculated considering the following values: $S_{\mathrm{S}}=4000 \mathrm{MVA}, \quad S_{\mathrm{R}}=50 \mathrm{MVA}, \quad E_{1 \mathrm{R}}=E_{\mathrm{L} N}, \quad E_{\mathrm{sc}}=8 \%\left(E_{1 \mathrm{R}}\right)$, $S_{\mathrm{L}}=S_{\mathrm{L} 1}+S_{\mathrm{L} 2}=50 \mathrm{MW}$. With this set of data and the mentioned base values, the impedances in the circuit result: $z_{\mathrm{S}}=0.0375 \mathrm{p} . \mathrm{u}$. and $z_{\mathrm{T}}=0.08 \mathrm{p} \cdot \mathrm{u}$.

\subsection{Simulation results}

The three connection options in Figs. $7 b-d$ have been tested under different load unbalance conditions, which are obtained by changing the share of the total power $S_{\mathrm{L}}$ on the two phases

$$
S_{\mathrm{L} 1}=k \cdot S_{\mathrm{L}} ; \quad S_{\mathrm{L} 2}=S_{\mathrm{L}}-S_{\mathrm{L} 1}=(1-k) \cdot S_{\mathrm{L}}
$$

The phase voltage results are reported in Table 1 for each connection, considering three cases of load unbalance $(k=0, k=$ $0.5, k=1)$. The corresponding voltage phasors are depicted in Fig. 8, where the voltage shifting of the neutral point on the secondary side can be observed under the considered topologies: its deviation, in absolute, decreases from about 0.5 p.u. with the single-phase connection to about 0.35 p.u. with the Scott transformer. 
Table 2 Voltage unbalance on the primary side of the transformer both graphically obtained from [31] and evaluated through the proposed generalised methodology. The relative error $\delta$ is reported for each configuration

\begin{tabular}{|c|c|c|c|c|c|c|c|c|c|}
\hline \multirow[t]{2}{*}{$\bar{k}$} & \multicolumn{3}{|c|}{ 1-ph } & \multicolumn{3}{|c|}{ V-conn } & \multicolumn{3}{|c|}{ Scott } \\
\hline & $\begin{array}{c}\text { Chen and Kuo } \\
\text { [31], \% }\end{array}$ & $\begin{array}{c}\text { Simulation } \\
\%\end{array}$ & $\begin{array}{l}\delta \\
\%\end{array}$ & $\begin{array}{c}\text { Chen and Kuo } \\
\text { [31], \% }\end{array}$ & $\begin{array}{c}\text { Simulation } \\
\%\end{array}$ & $\begin{array}{l}\delta \\
\%\end{array}$ & $\begin{array}{c}\text { Chen and Kuo } \\
\text { [31] \% }\end{array}$ & $\begin{array}{c}\text { Simulation } \\
\%\end{array}$ & $\begin{array}{c}\delta \\
\%\end{array}$ \\
\hline$\overline{0}$ & 1.244 & 1.245 & 0.08 & 1.244 & 1.245 & 0.08 & 1.244 & 1.245 & 0.08 \\
\hline 0.1 & 1.244 & 1.245 & 0.08 & 1.055 & 1.057 & 0.19 & 0.995 & 0.997 & 0.20 \\
\hline 0.2 & 1.244 & 1.245 & 0.08 & 0.890 & 0.887 & -0.34 & 0.747 & 0.748 & 0.13 \\
\hline 0.3 & 1.244 & 1.245 & 0.08 & 0.750 & 0.747 & -0.40 & 0.498 & 0.499 & 0.20 \\
\hline 0.4 & 1.244 & 1.245 & 0.08 & 0.655 & 0.653 & -0.31 & 0.249 & 0.249 & 0.00 \\
\hline 0.5 & 1.244 & 1.245 & 0.08 & 0.625 & 0.624 & -0.16 & 0.000 & 0.000 & 0.00 \\
\hline 0.6 & 1.244 & 1.245 & 0.08 & 0.670 & 0.668 & -0.30 & 0.250 & 0.249 & -0.40 \\
\hline 0.7 & 1.244 & 1.245 & 0.08 & 0.769 & 0.771 & 0.26 & 0.501 & 0.499 & -0.40 \\
\hline 0.8 & 1.244 & 1.245 & 0.08 & 0.910 & 0.910 & 0.00 & 0.751 & 0.748 & -0.40 \\
\hline 0.9 & 1.244 & 1.245 & 0.08 & 1.070 & 1.072 & 0.19 & 0.998 & 0.997 & -0.10 \\
\hline 1 & 1.244 & 1.245 & 0.08 & 1.244 & 1.245 & 0.08 & 1.244 & 1.245 & 0.08 \\
\hline
\end{tabular}

Finally, the voltage unbalance on the primary side, in terms of ratio between the negative and positive sequence voltage components, is shown in Fig. 9 for the three considered topologies and different values of $k$. It is confirmed that, as expected, the Scott connection is the only one which allows to eliminate the voltage unbalance when the two single phase loads are equal $(k=0.5)$. The results shown here are consistent with the ones in [31] which are achieved with a different methodology and therefore validate the general applicability of the approach proposed in this work.

The results in terms of voltage unbalance are reported in Table 2 to show the consistency with those in [31], from which they are derived by graphical processing. It can be seen that the maximum error reaches $0.4 \%$, while in average it is $0.01 \%$.

\section{Conclusions}

This paper introduces a generalised modelling methodology for multi-circuit multi-phase transformers in power systems. The aim of this work is the formalisation of a flexible approach in modelling the transformer and allowing any kind of topology to be straightforwardly represented. In particular, the developed method is readily applicable in solving the power flow problem in a multiconductor frame thus allowing a high detail level in the power system models.

The general approach is initially applied to a three-phase twowinding transformer and then generalised to consider any number of windings (three in the example). The zig-zag and Scott connections are used to describe its applicability to special topologies by simply adopting an incidence matrix approach. In this way, the inclusion of models with uneven number of phases on the respective transformer sides is allowed. As a result, the developed method could help the investigation about the interaction among power systems at different voltage levels. Furthermore, the matrix approach facilitates the scalability, straightforward implementation in computer simulations and easy application to big networks.

The developed methodology has been validated by comparing results with the literature. So power flow solution methods (for instance current injection solvers) can make use of the proposed approach to model generic transformers, coupling multiple systems with different main characteristics in terms of rated voltages, phase number and phase voltage displacement.

\section{References}

[1] Gestore Serivizi Energetici (GSE): 'Statistical report on solar photovoltaic' 2015, in Italian, available at: http://www.gse.it/it/Statistiche/ RapportiStatistici/

[2] ENTSO-E: 'Consumption data statistics, hourly values'. Available at: www.entsoe.eu/data/data-portal/consumption/

[3] Coppo, M., Pelacchi, P., Pilo, F., et al.: 'The Italian smart grid pilot projects: selection and assessment of the test beds for the regulation of smart electricity distribution', Electr. Power Syst. Res., 2015, 120, pp. 136-149, 10.1016/ j.epsr.2014.06.018
[4] Madureira, A.G., Lopes, J.A.P.: 'Ancillary services market framework for voltage control in distribution networks with microgrids', Electr. Power Syst. Res., 2012, 86, pp. 1-7, 10.1016/j.epsr.2011.12.016

[5] Petretto, G., Cantù, M., Gigliucci, G., et al.: 'Representative distribution network models for assessing the role of active distribution systems in bulk ancillary services markets'. 19th Power Systems Computation Conf., PSCC 2016, 2016, 10.1109/pscc.2016.7541020

[6] Caldon, R., Coppo, M., Turri, R.: 'Voltage unbalance compensation in LV networks with inverter interfaced distributed energy resources'. 2012 IEEE Int. Energy Conf. and Exhibition (ENERGYCON), 2012, pp. 527-532, 10.1109/energycon.2012.6348210

[7] Bracale, A., Caldon, R., Coppo, M., et al:: 'Active management of distribution networks with the Atlantide models'. 8th Mediterranean Conf Power Generation, Transmission, Distribution and Energy Conversion (MEDPOWER 2012), October 2012, pp. 1-7, 10.1049/cp.2012.2062

[8] Caldon, R., Coppo, M., Turri, R.: 'Coordinated voltage control in MV and LV distribution networks with inverter-interfaced users'. 2013 IEEE Grenoble Conf. PowerTech, POWERTECH 2013, 2013, pp. 1-5, 10.1109/ ptc.2013.6652491

[9] Savio, A., Bignucolo, F., Caldon, R.: 'Contribution of MV static distributed generation to voltage unbalance mitigation'. 2016 AEIT Int. Annual Conf., Capri, France, October 2016, DOI: 10.23919/AEIT.2016.7892739

[10] Vandoorn, T., De Kooning, J.D.M., Meersman, B. et al.: 'Voltage-based control of a smart transformer in a microgrid', IEEE Trans. Ind. Electron. 2013, 60, (4), pp. 1291-1305, 10.1109/tie.2011.2165463

[11] Coppo, M., Turri, R., Marinelli, M., et al.: 'Voltage management in unbalanced low voltage networks using a decoupled phase-tap-changer transformer', 2014 49th Int. Universities Power Engineering Conf. (UPEC), 2014, pp. 1-6, 10.1109/upec.2014.6934809

[12] Hu, J., Marinelli, M., Coppo, M., et al.: 'Coordinated voltage control of a decoupled three-phase on-load tap changer transformer and photovoltaic inverters for managing unbalanced networks', Electr. Power Syst. Res., 2016 , 131, pp. 264-274, 10.1109/upec. 2015.7339880

[13] Yun, L., Crossley, P.A.: 'Voltage balancing in low-voltage radial feeders using Scott transformers', IET. Gener. Transm. Distrib., 2014, 8, (8), pp. 1489 1498, 10.1049/iet-gtd.2013.0528

[14] Jou, H.L., Wu, J.C., Chiang, W.J., et al: 'Analysis of zig-zag transformer applying in the three-phase four-wire distribution power system', IEEE Trans. Power Deliv., 2005, 20, (2), pp. 1168-1173, 10.1109/tpwrd.2005.844281

[15] Alepuz, S., Gonzalez, F., Martin-Arnedo, J., et al.: 'Solid state transformer with low-voltage ride-through and current unbalance management capabilities'. Industrial Electronics Society, IECON 2013 - 39th Annual Conf. of the IEEE, 2013, pp. 1278-1283, 10.1109/iecon.2013.6699316

[16] Bignucolo, F., Bertoluzzo, M., Fontana, C.: 'Applications of the solid state transformer concept in the electrical power system'. 2015 AEIT Int. Annual Conf., AEIT, 2015, 10.1109/aeit.2015.7415235

[17] Guerra, G., Martinez-Velasco, J. A.: 'A solid state transformer model for power flow calculations', Int. J. Electr. Power Energy Syst., 2017, 89, pp. 4051, 10.1016/j.ijepes.2017.01.005

[18] Martinez, J.A., Mahseredjian, J.: 'Load flow calculations in distribution systems with distributed resources. A review'. IEEE Power and Energy Society General Meeting, 2011, pp. 1-8, 10.1109/pes.2011.6039172

[19] Chen, T.H., Chen, M.-S., Hwang, K.J., et al.: 'Distribution system power flow analysis - a rigid approach', Power Deliv., 1991, 6, (3), pp. 1146-1152, $10.1109 / 61.85860$

[20] Chen, T.-H., Yang, N.-C.: 'Loop frame of reference based three-phase power flow for unbalanced radial distribution systems', Electr. Power Syst. Res., 2010, 80, (7), pp. 799-806, 10.1016/j.epsr.2009.12.006

[21] Vieira, J.C.M., Freitas, W., Morelato, A.: 'Phase-decoupled method for threephase power-flow analysis of unbalanced distribution systems', IEE Proc. Gener. Transm. Distrib., 2004, 151, (5), pp. 568-574, 10.1049/ipgtd:20040831

[22] Dugan, R.C., McDermott, T.E.: 'An open source platform for collaborating on smart grid research'. Power and Energy Society General Meeting, 2011, 2011, pp. $1-7,10.1109 /$ pes.2011.6039829 
[23] Arboleya, P., Gonzalez-Moran, C., Coto, M.: 'Unbalanced power flow in distribution systems with embedded transformers using the complex theory in stationary reference frame', IEEE Trans. Power Syst., 2014, 29, (3), pp. 1012-1022, 10.1109/tpwrs. 2013.2292112

[24] Abdel-Akher, M., Nor, K.M., Rashid, A.H.A.: 'Improved three-phase powerflow methods using sequence components', IEEE Trans. Power Syst., 2005, 20, (3), pp. 1389-1397, 10.1109/tpwrs.2005.851933

[25] Dafic, I., Jabr, R.A., Neisius, H.T. 'Transformer modeling for three-phase distribution network analysis', IEEE Trans. Power Syst., 2015, 30, (5), pp. 2604-2611, 10.1109/TPWRS.2014.2360812

[26] Chen, T.H., Chang, J.D.: 'Open wye-open delta and open delta-open delta transformer models for rigorous distribution system analysis', IEE Proc. C Gener. Transm. Distrib., 1992, 139, (3), pp. 227-234, 10.1049/ip-c.1992.0035

[27] del Vecchio, R. M.: 'Multiterminal three phase transformer model with balanced or unbalanced loading', IEEE Trans. Power Deliv., 2008, 23, (3), pp. 1439-1447, 10.1109/tpwrd.2007.916229

[28] Moorthy, S.S., Hoadley, D.: 'A new phase-coordinate transformer model for ybus analysis', IEEE Trans. Power Syst., 2002, 17, (4), pp. 951-956, 10.1109/ tpwrs.2002.804996

[29] Dugan, R.C., Santoso, S.: 'An example of 3-phase transformer modeling for distribution system analysis'. Proc. IEEE Power Engineering Society Transmission and Distribution Conf., 2003, vol. 3, pp. 1028-1032, 10.1109/ tdc.2003.1335084

[30] Kocar, I., Lacroix, J.S.: 'Implementation of a modified augmented nodal analysis based transformer model into the backward forward sweep solver', IEEE Trans. Power Syst., 2012, 27, (2), pp. 663-670, 10.1109/ TPWRS.2011.2175256

[31] Chen, T.H., Kuo, H.Y.: 'Network modelling of traction substation transformers for studying unbalance effects', IEE Proc., Gener. Transm. Distrib., 1995, 142, (2), pp. 103-108, 10.1049/ip-gtd:19951592

[32] Azizian, D.: 'A new multi-winding traction transformer equivalent circuit for short-circuit performance analysis', Int. Trans. Electr. Energy Syst., 2014, 24, pp. 186-202, 10.1002/etep.1686

[33] Sunderland, K., Coppo, M., Conlon, M., et al.: 'A correction current injection method for power flow analysis of unbalanced multiple-grounded 4-wire distribution networks', Electr. Power Syst. Res., 2016, 132, pp. 30-38, 10.1016/j.epsr.2015.10.027

[34] Wang, X., Song, Y., Irving, M.: 'Modern Power Systems Analysis' (Springer, USA, 2010), ISBN: 9780387728537

[35] IEEE: 'Distribution test feeders'. Available at: https://ewh.ieee.org/soc/pes/ dsacom/testfeeders/

\section{Appendix. Validation with IEEE-4 bus feeder}

To better validate the model, it has been employed in the IEEE-4 bus feeder, which is designed to test the representation of threephase two-winding transformers in the presence of balanced and unbalanced loads [35]. The test circuit is composed by four buses with two voltage levels: $12.47 \mathrm{kV}$ for Buses 1 (slack bus) and 2; $4.16 \mathrm{kV}$ for Buses 3 and 4.

In the following, the methodology discussed in this paper is applied to the open-wye-open-delta transformer. Referring to the connection scheme shown in [35], only two single-phase circuits $\left(n_{\mathrm{c}}=2\right)$ are needed for this specific case. The primitive matrix $\boldsymbol{Y}_{\mathbf{P}(\mathbf{0})}$ in (28) is calculated in p.u. with respect to a base power of 1 MVA and base line-line voltages 12.47 and $4.16 \mathrm{kV}$, using the following data (referred to each single-phase circuit in the step-down case):

- Primary and secondary windings rated voltages: 7.2 and 4.16 $\mathrm{kV}$.
- Rated power: 2 MVA.

- Short-circuit impedance $\dot{z}_{n_{\mathrm{w}}}=1+\mathrm{j} 6[\%]$.

- Iron core and magnetisation losses are neglected since no reference is given in [35]: very low value is considered for the open-circuit current (i.e. $i_{0}=10^{-6} \mathrm{p}$.u. with respect to the nominal feeding current) (see (28))

Following the method discussed in Section 2, the matrices used in this case are shown in (29)-(32) leading to the transformer matrix in (33). From (32), it can be seen that the number of ports is kept to four on both sides for simplicity while using four-wire lines

$$
\boldsymbol{A}_{(0)}=\left[\begin{array}{cccccc}
1 & 0 & 0 & 0 & -1 & 0 \\
0 & 1 & 0 & 0 & 0 & -1 \\
0 & 0 & 1 & 0 & -1 & 0 \\
0 & 0 & 0 & 1 & 0 & -1 \\
0 & 0 & 0 & 0 & 1 & 0 \\
0 & 0 & 0 & 0 & 0 & 1
\end{array}\right]
$$

(see (30))

$$
\begin{gathered}
\boldsymbol{M}=\left[\begin{array}{ccccc}
1 & 0 & 0 & 0 \\
0 & 1 & 0 & 0 \\
0 & 0 & 0.577 & 0 \\
0 & 0 & 0 & 0.577
\end{array}\right] \\
\boldsymbol{C}=\left[\begin{array}{cccccccc}
1 & 0 & 0 & -1 & 0 & 0 & 0 & 0 \\
0 & 1 & 0 & -1 & 0 & 0 & 0 & 0 \\
0 & 0 & 0 & 0 & 1 & -1 & 0 & 0 \\
0 & 0 & 0 & 0 & 0 & 1 & -1 & 0
\end{array}\right]
\end{gathered}
$$

(see (33)) To ease the comparison, node voltage results for the IEEE-4 bus feeder are reported from [35] in Table 3 considering a step-down transformer, unbalanced loading and four connection options. The results obtained with the proposed method for the transformer modelling are reported in Table 4, where it can be seen that they are in good agreement with those published in [35]. The error, in fact, is below $0.5 \%$ (which is indicated as expected maximum deviation) with an average of $0.08 \%$. It is worth noting that the reported error just refers to the voltage magnitude since the results in terms of voltage angle are perfectly matching up to the first decimal place (which is the precision in the reference results).

$$
\boldsymbol{Y}_{\mathbf{P}(0)}=\left[\begin{array}{cccccc}
10.811-\mathrm{j} 64.865 & 0 & 0 & 0 & 0 & 0 \\
0 & 10.811-\mathrm{j} 64.865 & 0 & 0 & 0 & 0 \\
0 & 0 & 10.811-\mathrm{j} 64.865 & 0 & 0 & 0 \\
0 & 0 & 0 & 10.811-\mathrm{j} 64.865 & 0 & 0 \\
0 & 0 & 0 & 0 & 2 \times 10^{-6} & 0 \\
0 & 0 & 0 & 0 & 0 & 2 \times 10^{-6}
\end{array}\right]
$$

$$
\boldsymbol{Y}_{\mathbf{W}}=\left[\begin{array}{cccc}
5.405-\mathrm{j} 32.432 & 0 & -5.405+\mathrm{j} 32.432 & 0 \\
0 & 5.405-\mathrm{j} 32.432 & 0 & -5.405+\mathrm{j} 32.432 \\
-5.405+\mathrm{j} 32.432 & 0 & 5.405-\mathrm{j} 32.432 & 0 \\
0 & -5.405+\mathrm{j} 32.432 & 0 & 5.405-\mathrm{j} 32.432
\end{array}\right]
$$




$$
\left[\begin{array}{cccccccc}
5.405-\mathrm{j} 32.432 & 0 & 0 & -5.405+\mathrm{j} 32.432 & -3.121+\mathrm{j} 18.725 & 3.121-\mathrm{j} 18.725 & 0 & 0 \\
0 & 5.405-\mathrm{j} 32.432 & 0 & -5.405+\mathrm{j} 32.432 & 0 & -3.121+\mathrm{j} 18.725 & 3.121-\mathrm{j} 18.725 & 0 \\
0 & 0 & 0 & 0 & 0 & 0 & 0 & 0 \\
-5.405+\mathrm{j} 32.432 & -5.405+\mathrm{j} 32.432 & 0 & 10.811-\mathrm{j} 64.865 & 3.121-\mathrm{j} 18.725 & 0 & -3.121+\mathrm{j} 18.725 & 0 \\
-3.121+\mathrm{j} 18.725 & 0 & 0 & 3.121-\mathrm{j} 18.725 & 1.802-\mathrm{j} 10.811 & -1.802+\mathrm{j} 10.811 & 0 & 0 \\
3.121-\mathrm{j} 18.725 & -3.121+\mathrm{j} 18.725 & 0 & 0 & -1.802+\mathrm{j} 10.811 & 3.604-\mathrm{j} 21.622 & -1.802+\mathrm{j} 10.811 & 0 \\
0 & 3.121-\mathrm{j} 18.725 & 0 & -3.121+\mathrm{j} 18.725 & 0 & -1.802+\mathrm{j} 10.811 & 1.802-\mathrm{j} 10.811 & 0 \\
0 & 0 & 0 & 0 & 0 & 0 & 0 & 0
\end{array}\right]
$$

Table 3 Reference node voltages for the IEEE-4 bus feeder with step-down transformer and unbalanced loading [35]

\begin{tabular}{|c|c|c|c|c|c|c|c|c|c|}
\hline \multirow[t]{2}{*}{ Bus } & \multirow[t]{2}{*}{ phase number } & \multicolumn{2}{|c|}{ Gr. Y- } & \multicolumn{2}{|c|}{$\Delta-$ Gr. Y } & \multicolumn{2}{|c|}{$\Delta-\Delta$} & \multicolumn{2}{|c|}{ Open Gr. Y-s } \\
\hline & & Mag., V & Angle, $\angle{ }^{\circ}$ & Mag., V & Angle, $\angle \circ$ & Mag., V & Angle, $\angle^{\circ}$ & Mag., V & Angle, $\angle^{\circ}$ \\
\hline \multirow[t]{3}{*}{2} & 1 & 7113 & $\angle-0.2$ & 12,350 & $\angle 29.6$ & 12,341 & $\angle 29.8$ & 6952 & $\angle 0.7$ \\
\hline & 2 & 7144 & $\angle-120.4$ & 12,314 & $\angle-90.4$ & 12,370 & $\angle-90.5$ & 7172 & $\angle-122$ \\
\hline & 3 & 7111 & $\angle 119.5$ & 12,333 & $\angle 149.8$ & 12,302 & $\angle 149.5$ & 7313 & $\angle 120.5$ \\
\hline \multirow[t]{3}{*}{3} & 1 & 3896 & $\angle-2.8$ & 2290 & $\angle-32.4$ & 3902 & $\angle 27.2$ & 3632 & $\angle 0.1$ \\
\hline & 2 & 3972 & $\angle-123.8$ & 2261 & $\angle-153.8$ & 3972 & $\angle-93.9$ & 4121 & $\angle-127.6$ \\
\hline & 3 & 3875 & $\angle 115.7$ & 2214 & $\angle 85.2$ & 3871 & $\angle 145.7$ & 3450 & $\angle 108.9$ \\
\hline \multirow[t]{3}{*}{4} & 1 & 3425 & $\angle-5.8$ & 2157 & $\angle-34.2$ & 3431 & $\angle 24.3$ & 3307 & $\angle-1.5$ \\
\hline & 2 & 3646 & $\angle-130.3$ & 1936 & $\angle-157$ & 3647 & $\angle-100.4$ & 3907 & $\angle-131.9$ \\
\hline & 3 & 3298 & $\angle 108.6$ & 1849 & $\angle 73.4$ & 3294 & $\angle 138.6$ & 3073 & $\angle 103.1$ \\
\hline
\end{tabular}

Table 4 Node voltages for the IEEE-4 bus feeder computed with the proposed method. Relative error $\delta$ between the obtained results and [35] are reported in \%o

\begin{tabular}{|c|c|c|c|c|c|c|c|c|c|c|c|c|c|}
\hline \multirow[t]{2}{*}{ Bus } & \multirow[t]{2}{*}{ phase number } & \multicolumn{3}{|c|}{ Gr.Y- } & \multicolumn{2}{|c|}{$\Delta-$ Gr.Y } & \multirow[b]{2}{*}{$\delta, \%$} & \multicolumn{3}{|c|}{$\Delta-\Delta$} & \multicolumn{3}{|c|}{ Open Gr.Y- $\Delta$} \\
\hline & & Mag., V & Angle $\angle^{\circ}$ & $\delta, \%$ & Mag., V & Angle, $\angle^{\circ}$ & & Mag., V & Angle, $\angle^{\circ}$ & $\delta, \%$ & Mag., V & Angle, $\angle^{\circ}$ & $\delta, \%$ \\
\hline \multirow[t]{3}{*}{2} & 1 & 7113 & $\angle-0.2$ & 0.00 & 12,350 & $\angle 29.6$ & 0.00 & 12,341 & $\angle 29.8$ & 0.00 & 6952 & $\angle 0.7$ & 0.00 \\
\hline & 2 & 7143 & $\angle-120.4$ & -0.14 & 12,314 & $\angle-90.4$ & 0.00 & 12,370 & $\angle-90.5$ & 0.00 & 7172 & $\angle-122$ & 0.00 \\
\hline & 3 & 7110 & $\angle 119.5$ & -0.14 & 12,333 & $\angle 149.8$ & 0.00 & 12,302 & $\angle 149.5$ & 0.00 & 7313 & $\angle 120.5$ & 0.00 \\
\hline \multirow[t]{3}{*}{3} & 1 & 3896 & $\angle-2.8$ & 0.00 & 2290 & $\angle-32.4$ & 0.00 & 3902 & $\angle 27.2$ & 0.00 & 3633 & $\angle 0.1$ & 0.28 \\
\hline & 2 & 3972 & $\angle-123.8$ & 0.00 & 2262 & $\angle-153.8$ & 0.44 & 3973 & $\angle-93.9$ & 0.25 & 4121 & $\angle-127.6$ & 0.00 \\
\hline & 3 & 3875 & $\angle 115.7$ & 0.00 & 2214 & $\angle 85.2$ & 0.00 & 3872 & $\angle 145.7$ & 0.26 & 3451 & $\angle 108.9$ & 0.29 \\
\hline \multirow[t]{3}{*}{4} & 1 & 3426 & $\angle-5.8$ & 0.29 & 2157 & $\angle-34.2$ & 0.00 & 3431 & $\angle 24.3$ & 0.00 & 3308 & $\angle-1.5$ & 0.30 \\
\hline & 2 & 3647 & $\angle-130.3$ & 0.27 & 1936 & $\angle-157$ & 0.00 & 3648 & $\angle-100.4$ & 0.27 & 3907 & $\angle-131.9$ & 0.00 \\
\hline & 3 & 3298 & $\angle 108.6$ & 0.00 & 1849 & $\angle 73.4$ & 0.00 & 3294 & $\angle 138.6$ & 0.00 & 3074 & $\angle 103.1$ & 0.33 \\
\hline
\end{tabular}

